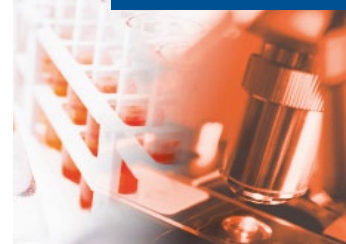

Berichte aus der Pharmaindustrie

Hypoxische Wunden

\title{
Mehr Sauerstoff für den Wundgrund
}

- Trotz einer adäquaten Behandlung der Primärerkrankungen wie chronisch venöser Insuffizienz, PAVK oder Diabetes mellitus und einer modernen feuchten Wundversorgung stagniert bei vielen Patienten mit chronischen Wunden der Heilungsprozess. Einer der Gründe: In den Wunden mangelt es an gewebeverfügbarem Sauerstoff. Wie Dr. Peter Engels, Molekularbiologe aus Bergisch Gladbach erläuterte, ist die Sauerstoffversorgung des Wundgrunds in der Regel bereits durch die Grunderkrankung „von innen“ her eingeschränkt. Gleichzeitig bestehe aber bei allen Prozessen der Wundheilung ein erhöhter Bedarf an Sauerstoff - sei es für die Keimabwehr durch reaktive Sauerstoffmoleküle, die Bildung von Granulationsgewebe, die Gefäßneubildung oder für den Aufbau eines stabilen Narbengewebes durch Kollagenreifung. Auch von außen gelangt nur wenig Sauerstoff zu den proliferierenden Zellen des Wundgrunds. Denn Wundexsudat stellt für den schlecht löslichen Sauerstoff ein kaum überwindbares Diffusionshindernis dar.

Ein jetzt auch in Deutschland verfügbares Hämoglobinspray (Granulox ${ }^{\circledast}$ ) kann die Wundheilung von außen durch eine verbesserte Sauerstoffversorgung unterstützen. Auf die gereinigte Wunde aufgesprüht, bindet das wasserlösliche Hämoglobin Umgebungssauerstoff und gibt ihn am Wundgrund wieder $a b$, erklärte Michael Sander vom Unternehmen SastoMed den Wirkmechanismus. Nach dem Prinzip der erleichterten Diffusion könne der Sauerstoff mithilfe des Transportmoleküls so auch das Wundexsudat durchdringen. Wie Interimsergebnisse einer Anwendungsstudie in Prag zeigen, trägt das Spray zu einer deutlichen Beschleunigung der Wundheilung bei, so Sander.

Ergänzend stellte Nesat Mustafi Fallbeispiele zur praktischen Anwendung des Hämoglobinsprays vor. Der Pflegerische Leiter der Wundambulanz Frankfurt/Main Nordwest setzt das Spray bei therapierefraktären Patienten mit zum Teil langjährigen chronischen Wunden ein. Bei allen behandelten Wunden konnte er nach kurzer Zeit ein Fortschreiten der Wundheilung beobachten. Bei vielen Patienten kam es erstmalig nach Jahren zum Wundverschluss. ne

Quelle: Satellitensymposium, 6. Deutscher Wundkongress, 10.5.2012, Bremen, Veranstalter: SastoMed GmbH

\section{Diabetiker mit kardiovaskulären Risiken Blutzuckerschwankungen sind Gift für das Herz}

- Diabetiker sterben häufig an kardiovaskulären Komplikationen. Und bei mehr als 50\% aller Patienten mit Herzinfarkt findet sich entweder ein Diabetes oder eine Störung der Glukosetoleranz. Deshalb empfehlen kardiologische und diabetologische Leitlinien, bei Diabetikern nach Herzkrankheiten und bei Herzkranken nach Diabetes zu fahnden, erläuterte Dr. Anselm Gitt, Ludwigshafen.

Wenn kardiovaskuläre Risiken bei Diabetikern frühzeitig erkannt werden, kann die diabetische Kardiomyopathie häufig noch aufgehalten werden, so Gitt. Im Rahmen eines ganzheitlichen Therapieprinzips müssen dabei alle kardiovaskulären Risikofaktoren wie Cholesterin, Blutdruck, Rauchen, Übergewicht oder Bewegungsarmut angegangen werden. Auch die antidiabetische Therapie spielt eine entscheidende Rolle. Starke Blutzuckerschwankungen und Hypoglykämien sind Gift für das Herz, weil sie kardiale Ischämien sowie Herzrhythmusstörungen begünstigen, berichtete Gitt.

\section{Analoginsuline wirken kardioprotektiv}

Moderne Analoginsuline haben hier Vorteile gegenüber konventionellen Insulinen: Kurzwirksame Analoginsuline kopieren die natürliche Insulinwirkung besser, langwirksame Analoginsuline sorgen für konstantere Basalinsulinspiegel im Tagesverlauf. Zudem sind Fehler beim Mischen, wie sie bei NPH-Insulin auftreten können, nicht möglich, erläuterte der Diabetologe Dr. Marcel Kaiser. In sieben verschiedenen Studien konnte gezeigt werden, dass eine Therapie mit Insulindetemir (Levemir ${ }^{\circledR}$ ) im Vergleich zu NPHInsulin das relative Hypoglykämierisiko jeweils statistisch signifikant um 26-65\% senkt.

Auf kardioprotektive Effekte der Analoginsuline deuten auch die Ergebnisse einer prospektiven Studie mit 104 Typ-2-Diabetikern hin, die entweder mit Insulinaspart plus Insulindetemir oder mit Humaninsulin plus NPH-Insulin behandelt wurden: Nach 24 Monaten hatten die Patienten unter Analoginsulinen nicht nur deutlich bessere postprandiale Blutzuckerverläufe, sondern auch klinisch relevante Verbesserungen der systolischen und diastolischen Herzfunktion, erläuterte Studienautor Dr. Thorsten Siegmund, München. Gerade für den kardial gefährdeten Diabetiker bieten moderne Insuline deshalb Vorteile, so Siegmund.

red

Quelle: Verlagsworkshop „Kardioprotektion und moderne Insuline in der Diabetestherapie“, München, Oktober 2011 (unterstützt von Novo Nordisk). 